\title{
Manipulating the bioenergetics of alloreactive T cells causes their selective apoptosis and arrests graft versus host disease
}

\author{
Erin Gatza ${ }^{1, \ddagger}$, Daniel R. Wahl ${ }^{2, \ddagger}$, Anthony W. Opipari ${ }^{3}$, Thomas B. Sundberg ${ }^{4}$, Pavan \\ Reddy $^{5}$, Chen Liu ${ }^{6}$, Gary D. Glick ${ }^{2,4}$, and James L.M. Ferrara ${ }^{1}$ \\ ${ }^{1}$ Department of Pediatrics, University of Michigan Ann Arbor, Michigan 48109, USA \\ ${ }^{2}$ Chemical Biology Doctoral Program, University of Michigan Ann Arbor, Michigan 48109, USA \\ ${ }^{3}$ Department of Obstetrics and Gynecology, University of Michigan Ann Arbor, Michigan 48109, \\ USA
}

${ }^{4}$ Department of Chemistry, University of Michigan Ann Arbor, Michigan 48109, USA

${ }^{5}$ Department of Internal Medicine, University of Michigan Ann Arbor, Michigan 48109, USA

${ }^{6}$ Department of Pathology, University of Florida College of Medicine, Gainesville, USA

\begin{abstract}
Cells generate ATP by glycolysis and by oxidative phosphorylation (OXPHOS) (1,2). Despite the importance of having sufficient ATP available for the energy-dependent processes involved in immune activation, little is known about the metabolic adaptations that occur in vivo to meet the increased demand for ATP in activated and proliferating lymphocytes. We found that bone marrow (BM) cells proliferating after bone marrow transplantation (BMT) increased aerobic glycolysis but not OXPHOS, while T cells proliferating in response to alloantigens during graft versus host disease (GVHD) increased both aerobic glycolysis and OXPHOS. Metabolomic analysis of alloreactive T cells showed an accumulation of acylcarnitines consistent with changes in fatty acid oxidation. Alloreactive $\mathrm{T}$ cells also exhibited a hyperpolarized mitochondrial membrane potential $(\Delta \Psi \mathrm{m})$, increased superoxide production and decreased antioxidant levels, whereas proliferating BM cells did not. Bz-423, a novel small molecule inhibitor of the mitochondrial $\mathrm{F}_{1} \mathrm{~F}_{0}$-ATPase, selectively increased superoxide and induced the apoptosis of alloreactive T cells, which arrested established GVHD in several BMT models without affecting hematopoietic engraftment or lymphocyte reconstitution. These findings challenge the current paradigm that activated T cells meet their increased demands for ATP though aerobic glycolysis, and identify the possibility that bioenergetic and redox characteristics can be selectively exploited as a novel therapeutic strategy for immune disorders.
\end{abstract}

\section{Introduction}

Cells generate ATP both by aerobic glycolysis and by OXPHOS $(1,2)$. Most cells metabolize glucose to pyruvate via glycolysis and then oxidize pyruvate to $\mathrm{CO}_{2}$ in the mitochondria, generating the large majority of their ATP through OXPHOS $(1,2)$. In contrast, many cancer cells and lymphocytes activated in vitro preferentially convert pyruvate into lactate that is secreted from the cells rather than oxidize pyruvate in the mitochondria $(1,2)$. This process, known as aerobic glycolysis, yields only 2 ATP per molecule of glucose, compared to a maximum of 36 ATP when glycolysis is coupled to

\footnotetext{
Correspondence to: Gary D. Glick; James L.M. Ferrara.

₹These authors contributed equally to this work
} 
OXPHOS $(2,3)$. While it seems counterintuitive for cells to employ a low-efficiency pathway to produce ATP under conditions of high energy demand, it has been proposed that aerobic glycolysis produces the requisite reducing equivalents and biosynthetic substrates that are required for proliferation (2).

Hematopoietic stem cells (HSCs) rapidly proliferate following BMT and differentiate to regenerate multiple blood cell lineages (hematopoiesis) (4). Like many other proliferating cell types, HSCs increase aerobic glycolysis rather than OXPHOS to meet increased ATP demands (5). Donor T cells may also proliferate following allogeneic BMT in response to histocompatibility antigens that are expressed on host tissues. These alloreactive $\mathrm{T}$ cells cause GVHD, which negatively impacts survival following BMT, both directly as a result of damage to target organs, and indirectly as a result of the infectious complications of the immunosuppressive therapies used to modulate this highly morbid complication of BMT (6, 7). Allogeneic BMT is thus currently used only for life-threatening hematologic and immunologic disorders, and new approaches to treat GVHD are urgently needed.

In contrast to the glycolytic phenotype of proliferating HSCs, proliferating lymphocytes that mediate immune diseases like lupus exhibit increased OXPHOS and reduced anti-oxidant levels $(8,9)$. We reasoned that if the alloreactive T cells that cause GVHD displayed such an oxidative phenotype, modulation of their metabolism could offer a novel therapeutic approach. We found that alloreactive donor T cells in fact increased both OXPHOS and aerobic glycolysis, and appeared bioenergetically similar to the lymphocytes that mediate lupus. These pathogenic lymphocytes also displayed hyperpolarization of their $\Delta \Psi_{\mathrm{m}}$ and depleted antioxidant stores. We exploited these bioenergetic abnormalities with Bz-423, a small molecule that inhibits the mitochondrial $\mathrm{F}_{1} \mathrm{~F}_{0}$-ATPase and induces apoptosis that requires the generation of reactive oxygen species (ROS) $(10,11)$. Bz-423 treatment induced selective apoptosis of alloreactive donor T cells and reversed GVHD in several BMT models without affecting hematopoietic engraftment or immunologic reconstitution.

\section{Results}

\section{Bioenergetics of BM and T cells proliferating in vivo}

Following BMT, small numbers of HSCs rapidly expand to generate multiple hematopoietic lineages, including white blood cells, red blood cells, and platelets (4). We compared the bioenergetics of proliferating BM cells 8-9 days after their transplantation into lethally irradiated, syngeneic hosts to the bioenergetics of naïve BM cells (Fig. S1A). For each group of cells, we measured rates of lactate production (which reflects the rate of aerobic glycolysis), oligomycin-inhibited oxygen consumption (which reflects the rate of OXPHOS) (12-14) and the expression of GLUT1, the major glucose transporter on hematopoietic cells $(15,16)$. We observed that lactate production increased 3-fold and GLUT1 expression doubled in proliferating BM cells post transplant (PT) relative to naïve (N) BM cells (Figs. $1 \mathrm{~A}, 1 \mathrm{~B}$ and $\mathrm{S} 1 \mathrm{~B})$. By contrast, the rate of oxygen consumption in proliferating BM cells did not increase (Fig. 1C).

To examine the bioenergetics of lymphocytes that proliferate in response to alloantigens in vivo, we first analyzed T cells from a well-characterized model of GVHD in unirradiated recipients (B6 $\rightarrow$ B6D2F1) (17). Donor T cells isolated 7 days following transplant showed a 4-fold increase in lactate production and increased GLUT1 expression compared to naïve $\mathrm{T}$ cell controls (Figs 1D, E and S1C). These increases were similar to those observed in proliferating BM cells, and contrasted with a 40-80 fold increase in lactate production in mitogen-stimulated thymocytes $(18,19)$. 
The rate of oxygen consumption more than doubled in alloreactive $\mathrm{T}$ cells, contrasting with the basal rate observed in proliferating BM cells (Fig 1F). Oligomycin inhibited the same proportion (62-65\%) of oxygen consumption in all cell types tested, suggesting that they were similarly coupled $(20,21)$. Alloreactive lymphocytes proliferated faster than BM cells and lymphocytes proliferating after syngeneic BMT (Figs. 1G and S2). The faster proliferation and increased oxygen consumption in alloreactive $\mathrm{T}$ cells was associated with increased oxidative stress as indicated by a decrease in glutathione levels (Fig. S3).

The increased oxygen consumption in alloreactive T cells suggested increased TCA cycle activity and NADH production that could hyperpolarize $\Delta \Psi_{\mathrm{m}}$ (1). Donor T cells that did not proliferate to alloantigens remained detectable 4 days after the induction of GVHD in this model (Fig. S4), so we measured the mitochondrial activity in both proliferating and nonproliferating donor $\mathrm{T}$ cells with tetramethylrhodamine (TMRM), a dye that accumulates within mitochondria in proportion to $\Delta \Psi_{\mathrm{m}}$ (22). We observed increased TMRM fluorescence only in proliferating $\left(\mathrm{CFSE}^{\mathrm{lo}}\right)$ donor T cells (Figs. 1H and S5B) and in a small subpopulation of proliferating BM cells post-transplant (Figs. 1H and S6). This result is consistent with a recent study showing that the majority of hematopoetic stem cells have low rates of OXPHOS and depend on aerobic glycolysis for their energy needs (5). Since increased TMRM fluorescence in CFSE ${ }^{\text {lo }}$ donor T cells could be caused by increased mitochondrial number, we quantified mitochondrial mass with MitoTracker Green. Flourescence intensity did not change in proliferating $\mathrm{CD} 4^{+}$cells, and increased moderately in proliferating $\mathrm{CD}^{+}$cells. (Fig. S5C), showing that increased TMRM staining in alloreactive donor T cells is due, at least in part, to hyperpolarization of $\Delta \Psi_{\mathrm{m}}$.

To investigate further the unusual bioenergetic profile of alloreactive $\mathrm{T}$ cells, we compared levels of pyruvate, amino acids, and acylcarnitines of donor $\mathrm{T}$ cells isolated from the spleens of mice 7 days following induction of GVHD to levels in naïve donor T cells. As shown in Fig. 2A, intracellular levels of pyruvate in alloreactive $\mathrm{T}$ lymphocytes were almost undetectable. By contrast, the concentrations of amino acids changed minimally (Fig. 2B), whereas the concentrations of short-, medium- and long-chain acylcarnitines increased dramatically, with several species increasing $\geq 10$-fold (Fig. 2C). Acylcarnitines are obligate intermediates of fatty acids during their transport to the mitochondrial matrix where they are oxidized. Serum that was collected at the time of spleen harvest and analyzed separately showed that levels of fatty acids in mice with GVHD decreased compared to naïve donors $(0.43+0.01 \mathrm{mM}$ vs $0.55+0.02 \mathrm{mM} ; \mathrm{p}<0.05)$. These data suggest that the large increases of intracellular acylcarnitines resulted from intrinsic changes in cellular metabolism rather than from an increased supply of fatty acids in the circulation.

Because the observed mitochondrial changes can promote superoxide production from the mitochondrial respiratory chain (MRC) (23), we hypothesized that proliferating alloreactive $\mathrm{T}$ cells would also exhibit higher superoxide levels. We stained cells with dihydroethidium (DHE), a redox-sensitive dye specific for superoxide (24), and found that proliferating donor $\mathrm{CD}^{+}$and $\mathrm{CD} 8^{+} \mathrm{T}$ cells contained more superoxide than resting donor cells, whose superoxide remained at baseline (Figs. 3A and S5A).

\section{Bz-423 increases superoxide and causes selective apoptosis of alloreactive $T$ cells}

We hypothesized that the observed mitochondrial changes and reliance of proliferating donor T cells on OXPHOS would make them preferentially susceptible to agents that induce further oxidative stress by affecting the MRC. Bz-423, a benzodiazepine derivative that generates superoxide by inhibiting the mitochondrial $\mathrm{F}_{1} \mathrm{~F}_{0}$ - ATPase and inducing a state 3to-state 4 respiratory transition, is one such agent (25). A single Bz- 423 dose of $60 \mathrm{mg} / \mathrm{kg}$ (10\% of which is bioavailable) increased DHE and TMRM staining in proliferating donor $\mathrm{CD}^{+}$and $\mathrm{CD} 8^{+}$cells, but not in resting donor cells or host cells (Figs. 3A,B and S5A,B). 
Bz-423 also increased apoptosis in both $\mathrm{CD}^{+}$and $\mathrm{CD} 8^{+}$donor lymphocytes (Figs. 3C and $\mathrm{S} 5 \mathrm{D})$, but not in host $\mathrm{T}$ cells, donor and host $\mathrm{B}$ cells, or lymphocytes after syngeneic BMT (Fig. S7). Furthermore, Bz-423 did not increase DHE staining, TMRM staining, nor apoptosis in proliferating BM cells following syngeneic transplant (Figs 3D-F and S8).

\section{Bz-423 arrests ongoing GVHD by killing alloreactive T cells}

We next tested the therapeutic potential of Bz-423 in two allogeneic BMT models of GVHD. The first model (C3H.SW $\rightarrow$ B6-Ly5.2) is genetically similar to human leukocyte antigen (HLA)-identical, unrelated donor BMT (26), and GVHD is mediated by donor CD8 ${ }^{+}$ $\mathrm{T}$ cells responding to multiple minor histocompatibility antigens. We initiated Bz-423 treatment 7 days after BMT, by which time donor lymphocytes were already expanding, trafficking to target organs and initiating clinically evident disease (27). Bz- 423 treatment increased the apoptosis of proliferating, alloreactive $\mathrm{CD} 8^{+}$donor $\mathrm{T}$ cells, whereas other $\mathrm{T}$ cell populations, including those proliferating after syngeneic BMT, were unaffected (Figs. $4 \mathrm{~A}$ and S9). Six doses of Bz-423 over 2 weeks decreased by 4 -fold the population of donor $\mathrm{CD} 8^{+} \mathrm{T}$ cells that produce IFN- $\gamma$ and mediate GVHD in this model (Fig. 4B) (26). Bz-423 treatment also decreased the number of lymphocytes that infiltrated two primary GVHD target organs, the liver and the GI tract, and significantly reduced the overall damage in these organs (Fig. 4C,D and S10, S11). Bz-423 treatment significantly reduced GVHD clinical scores after 1 week (Fig. 4E), and improved survival compared to controls treated with vehicle ( $75 \%$ vs $29 \%$, p $<0.02$; Fig. 4E). We observed similarly improved survival when the treatment was continued for 10 weeks ( $74 \%$ vs $29 \%, \mathrm{p}=0.02)$. In another aggressive model of GVHD using a fully allogeneic donor/recipient strain combination (Balb/c $\rightarrow$ B6), Bz-423 treatment for 7 weeks again significantly reduced all clinical and histological parameters of disease (Fig. 4F, G).

Importantly, Bz-423 did not impair immunologic reconstitution in either the thymus or spleen following syngeneic BMT (Table S1), and all mice treated with drug showed complete donor BM engraftment. This favorable toxicity profile is consistent with other studies (28) and with the normal bioenergetic profile of rapidly proliferating BM cells [basal rates of oxygen consumption, normal levels of anti-oxidants and stable mitochondrial membrane potential] (Fig. 1). Bz-423 also did not impede the reconstitution of gastrointestinal mucosa by rapidly proliferating enterocytes following total body irradiation and BMT (Fig. S11). Thus Bz-423 affected only those proliferating cellular populations with chronically elevated mitochondrial respiration, in this case the alloreactive lymphocytes that mediate GVHD.

Bz-423 induces apoptosis through a redox-regulated process that is mediated by caspases $(10,11)$. To determine whether the arrest of GVHD by Bz- 423 required the apoptosis of alloreactive donor $\mathrm{T}$ cells, we injected transplanted mice with a pan-caspase inhibitor (quinolyl-valyl-O-methylaspartyl-[-2,6-difluorophenoxy]-methyl ketone; Q-VD-OPH) (29) 5 days after BMT and began treatment with Bz- 423 or vehicle 7 days after BMT. Q-VDOPH prevented the increase in apoptosis of activated $\mathrm{CFSE}{ }^{\text {lo }} \mathrm{CD} 8^{+}$donor $\mathrm{T}$ cells induced by Bz-423 (Fig. 4H) and abolished its ability to arrest GVHD (Fig. 4I). Injection of mice with Q-VD-OPH did not itself alter the kinetics of donor T cell proliferation (Fig. S12) or change the clinical course of GVHD (Fig. 4I).

\section{Discussion}

Hematopoietic cells of both myeloid and lymphoid lineages increase aerobic glycolysis to generate the ATP that is needed for them to proliferate in vitro (30). Although glycolysis generates ATP with significantly less efficiency than OXPHOS, it has been proposed that proliferating cells preferentially utilize aerobic glycolysis to provide both the biomass 
needed for the synthesis of macromolecules during cell division and the reducing equivalents needed to protect dividing cells from oxidative damage $(2,18,31)$. Notwithstanding the limitations imposed by certain assumptions, including similar coupling efficiencies between cellular populations and the potential derivation of lactate from alternative pathways, the rates of oxygen consumption and lactate production can be used to estimate the proportion of ATP derived from OXPHOS and aerobic glycolysis, respectively (13). Our results suggest that alloreactive T cells rely primarily on OXPHOS for their synthesis of ATP.

Carnitine is conjugated to acyl-CoA during the transport of fatty acids into the mitochondrial matrix where they undergo $\beta$-oxidation (1). The large increases in acylcarnitines, together with the extremely low levels of the glycolytic substrate pyruvate (Fig. 2A), and the GLUT1 transporter, suggests that fatty acids, rather than glucose, may be the principal substrates for ATP generation in these T cells. By comparison, the minimal changes in amino acids levels make them unlikely substrates for ATP generation. Although citrulline and ornithine are increased, neither amino acid is a direct substrate for ATP production, although both support the synthesis of nitric oxide (32) which increases in response to T cell activation (33).

Our findings challenge the current paradigm that activated $T$ cells satisfy their increased demand for energy through aerobic glycolysis $(1,2)$. The bioenergetic imbalances of alloreactive $\mathrm{T}$ cells result in the accumulation of acylcarnitines, excess superoxide, increased $\Delta \Psi_{\mathrm{m}}$, and diminished antioxidant reserves. While the basis for these changes has yet to be fully elucidated, they may be related to metabolic adaptations that occur as a result of chronic activation in the context of disease $(8,9)$. Similar changes have also been identified in other immune disorders and thus may represent a general feature of pathogenic $\mathrm{T}$ cells (9).

The oxidative phenotype of $\mathrm{T}$ cells that mediate GVHD provides a mechanistic basis for the specificity of Bz-423, a first-in-class $\mathrm{F}_{1} \mathrm{~F}_{0}$-ATP synthase inhibitor that eliminates the pathogenic cells and arrests the disease. Bz-423 has robust efficacy in several murine models of ongoing GVHD, where many immunosuppressive agents fail to show effects (34). Our data suggest that the efficacy and selectivity of Bz-423 derives from the convergence of its mechanism of action and the oxidative phenotype of alloreactive T cells. In contrast to either resting lymphocytes or proliferating bone marrow cells, alloreactive $\mathrm{T}$ cells are deficient in anti-oxidant reserves and generate additional superoxide in response to Bz-423, which leads to their apoptosis. The importance of endogenous antioxidants in modulating the effects of pro-oxidant drugs is well documented. For example, levels of intracellular antioxidants are inversely related to the apoptosis induced by arsenic trioxide, $\mathrm{N}-(4-$ hydroxyphenol) retinamide, paclitaxel and Bz-423 (35-38).

Our model predicts that Bz-423 does not generate superoxide or induce apoptosis in rapidly proliferating BM cells because these cells have normal bioenergetic phenotypes, with low rates of OXPHOS and significant antioxidant capacity. Likewise, Bz-423 does not impair the reconstitution of donor thymocytes, lymphocytes or granulocytes following syngeneic BMT for similar reasons. The specificity of Bz-423 for pathogenic lymphocytes, and its ability to preserve normal immune reconstitution, differentiates it from high dose systemic steroids, currently the standard treatment for $\operatorname{GVHD}(39,40)$. This mechanism of action is also consistent with the therapeutic benefits of $\mathrm{Bz}-423$ in models of autoimmune disease, where pathogenic cells have similar bioenergetic characteristics, increased respiration and depleted antioxidant reserves $(8,9)$. Recently, several groups have shown that mitochondrial metabolism can be pharmacologically manipulated during an immune response to promote the generation of memory cells and protective immunity $(41,42)$. While the mechanistic 
underpinnings of these findings have yet to be fully explored, it is clear that metabolismaltering drugs hold promise as immunotherapeutics in a broad spectrum of diseases.

\section{Materials and Methods}

Mice

Female C57B1/6 (B6:H-2 ${ }^{\mathrm{b}}, \mathrm{CD} 45.2^{+}$Thy 1.2 $2^{+}$), B6.Ly-5a (B6-Ly5.2:H-2 ${ }^{\mathrm{b}}$, CD45.1 $1^{+}$Thy $\left.1.2^{+}\right)$, and B6D2F1 (H-2 $2^{\text {bxd }}$, CD45.2 $2^{+}$Thy $1.2^{+}$) were purchased from Charles River Laboratories (Wilmington, MA). Female B6.PL-Thy 1a (B6-Thy1.1: H-2 ${ }^{\mathrm{b}}$, CD45. $2^{+}$Thy $\left.1.1^{+}\right)$and C3H.SW $\left(\mathrm{H}-2^{\mathrm{b}}, \mathrm{CD} 45.2^{+}\right)$were purchased from the Jackson Laboratory (Bar Harbor, ME). Female Balb/c $\left(\mathrm{H}-2^{\mathrm{d}}, \mathrm{CD} 45.2^{+}\right)$were purchased from Harlan Laboratories (Indianapolis, IN). All mice were at least 8 weeks of age prior to use and were cared for according to the Guidelines for Laboratory Animal Medicine at the University of Michigan.

\section{BMT}

To induce GVHD in non-irradiated recipients, syngeneic (B6) and allogeneic (B6D2F1) recipient mice were infused with $50.0 \times 10^{6}$ bulk splenocytes from B6-Ly5.2 or B6-Thy1.1 donor mice. B6-Ly5.2 mice were conditioned with a single dose of $900 \mathrm{cGy}$ TBI $\left({ }^{137} \mathrm{Cs}\right.$ source), followed by i.v. infusion of $5.0 \times 10^{6}$ bone marrow (BM) cells plus either $4.0 \times 10^{6}$ or $0.5 \times 10^{6}$ positively-selected $\mathrm{CD} 90^{+}$or $\mathrm{CD} 8^{+} \mathrm{T}$ cells, respectively, from allogeneic (C3H.SW) or syngeneic (B6) donor mice on day 0. B6 mice received a single dose of 1100 cGy TBI, followed by $5.0 \times 10^{6} \mathrm{BM}$ plus $5.0 \times 10^{6} \mathrm{CD} 90^{+} \mathrm{T}$ cells from B6 or Balb/c donor mice. In some experiments, donor splenocytes or T cells were labeled with CFDA SE (Vybrant CFDA SE Cell Tracer Kit; Invitrogen, Eugene, OR) per the manufacturer's protocol before their infusion. Bz-423 was administered i.p. in vehicle (2\% DMSO and 0.5\% carboxymethylcellulose in L-15 media) at a dose of $60 \mathrm{mg} / \mathrm{kg} 3$ times per week for 2-10 weeks, beginning 7 days after BMT. A single dose of Bz-423 was administered 4 or 7 days following BMT to measure apoptosis of donor T cells. Q-VD-OPH was diluted in DMSO and administered i.p. at a dose of $10 \mathrm{mg} / \mathrm{kg}$ (in $50 \mu \mathrm{L}$ ) on days 5-7 (for Annexin-V staining) or 5-19 (for survival experiments) after BMT. Administration of Q-VD-OPH was delayed by 5 days to avoid any suppressive effects on donor T cell activation. Reduced caspase activity ( $>68 \%$ ) was confirmed on day 7 using the ApoStat Intracellular Caspase Detection reagent (R\&D Systems, Minneapolis, MN). Transplanted mice were housed in sterile microisolator cages and given autoclaved hyperchlorinated $(\mathrm{pH}=3.0)$ drinking water for 3 weeks following BMT.

\section{Oxygen consumption, lactate measurements, and sample preparation for metabolomic analysis}

Bone marrow cells were flushed from the tibias and femurs of naïve or post-transplant mice using DMEM media and RBC-lysed prior to analysis. Unstimulated $\mathrm{T}$ cells were purified by positive selection using anti-CD90/Thy1.2 microbeads (Miltenyi Biotec) from spleens of B6 animals on the day of analysis. Donor (Thy 1.1) T cells were isolated from spleens of B6D2F1 animals 7 days after infusion of $50 \times 10^{6}$ B6-Thy1.1 splenocytes using a combination of PE-anti Thy 1.1 (BD Bioscience) and anti-PE microbeads (Miltenyi Biotec) and were typically $>80 \%$ pure. For metabolomic analyses, T cell pellets were sonicated in water and an aliquot was removed for protein quantification; the remainder was snap frozen in liquid nitrogen prior to analysis. Amino acids were analyzed by mass spectroscopy at the Stedman Nutrition \& Metabolism Center of Duke University. Acylcarnitine levels were analyzed by Lipomics Inc (Sacramento, CA). To measure oxygen consumption, cells were washed and resuspended at $2-5 \times 10^{6}$ cells $/ \mathrm{mL}$ in DMEM and analyzed at $37^{\circ} \mathrm{C}$ using an 
Oxygraph-2k oxygen electrode (Oroboros Instruments, Innsbruck, Austria). Oligomycin (Sigma, $1-2 \mu \mathrm{g} / \mathrm{mL}$ ) inhibited $60-70 \%$ of oxygen consumption in all samples.

To measure lactate production, cells were washed and resuspended at $5-15 \times 10^{6}$ cells $/ \mathrm{mL}$ in DMEM and aliquots were quenched at 4 time points over $2-3$ hours using $0.6 \mathrm{M}$ perchloric acid. After removal of cellular debris and neutralization with $\mathrm{NaOH}$, lactate concentrations at $>3$ time points were determined by incubating $10-20 \mu \mathrm{L}$ of sample with $1 \mu \mathrm{L}$ lactate dehydrogenase and $0.375 \mu \mathrm{L}$ glutamate-pyruvate transaminase (Sigma Aldrich) in 230-240 $\mu \mathrm{L}$ buffer containing $116 \mathrm{mM}$ glutamate and $0.96 \mathrm{mM} \mathrm{NAD}$ at $\mathrm{pH} 8.9$. Lactate levels were determined by monitoring absorption at $340 \mathrm{nM}$ and the rate of lactate production was calculated as a function of time and cell concentration (13).

\section{Pyruvate Measurements}

Cells were purified as for oxygen consumption experiments. Intracellular pyruvate levels were determined using the pyruvate assay kit (BioVision, Mountain View, CA).

\section{Analysis of GVHD}

Survival after BMT was monitored daily and clinical GVHD was assessed weekly using a previously described scoring system (43) which generates a composite GVHD score comprised of individual ratings for weight loss, posture, mobility, skin integrity and fur texture. Histopathological changes of GVHD were quantified in liver, skin and intestine, as described (43), by a single pathologist using coded slides.

\section{Flow cytometry}

Cells in single cell suspension were isolated from the spleens of transplanted mice. Cell surface staining was performed after $\mathrm{Fc}$ receptor blockade as previously described (44). $\Delta \Psi_{\mathrm{m}}$ and superoxide were measured using TMRM and DHE, respectively, as previously described $(10,11,25)$. TMRM and DHE data are plotted as the fold-increase in staining of gated donor and host $\mathrm{T}$ cell populations above donor and host cell staining in syngeneic controls. Annexin-V staining was performed in 1x Annexin Binding Buffer (BD Biosciences) containing cell surface antibodies and $1 \mathrm{~mL} /$ sample Annexin- $\mathrm{V}$ for 30 minutes on ice. The cells were washed and either resuspended in Annexin Binding Buffer for immediate analysis or fixed in PBS containing 4\% paraformaldehyde for 20 minutes on ice. IFN- $\gamma$ production by responder $\mathrm{CD}^{+} \mathrm{T}$ cells was assessed following in vitro restimulation with $2 \mu \mathrm{g} / \mathrm{mL}$ ionomycin and $50 \mathrm{ng} / \mathrm{mL}$ PMA for $5 \mathrm{~h}$ with or without Brefeldin A. To measure total cellular GLUT1, cells were surface stained and fixed, and after 1-2 days, permeabilized and incubated for 20 minutes with $10 \%$ normal mouse serum at $4{ }^{\circ} \mathrm{C}$. Cells were then incubated with rabbit anti-GLUT1 (Abcam) or isotype control for 45 minutes at $4^{\circ} \mathrm{C}$, washed twice, incubated with APC-labeled anti-Rabbit Fab (Jackson ImmunoResearch) for 45 minutes at $4{ }^{\circ} \mathrm{C}$, and washed twice more. All samples were analyzed using either a BD FACS Calibur cytometer and CellQuest software or a BD FACS Canto cytometer and FACSDiva software. FloJo software (TreeStar Inc., Ashland, OR) was used to calculate proliferation indices. Mitochondrial mass was assessed using MitoTracker Green as previously described (25). Antibodies used for flow cytometry can be found in Supplementary Material.

\section{Statistical Analysis}

Survival curves were plotted using Kaplan-Meier estimates and analyzed with the MantelCox log rank test. Student's T test and the Mann-Whitney U test were used for the statistical analysis of ex vivo data and clinical scores. $\mathrm{p}<0.05$ was considered statistically significant. 


\section{Supplementary Material}

Refer to Web version on PubMed Central for supplementary material.

\section{Acknowledgments}

We thank S. Clouthier, L. Swenson, for technical assistance, and P.L. Toogood for critical review of the manuscript. Supported by The Hartwell Foundation and NIH grants AI-047450 (G.D.G), P01 CA39542 (J.L.M.F.), T32 CA009676 and T32 CA88784-04 (E.G.). J.L.M.F. is an American Cancer Society Clinical Research Professor. E.G., D.R.W., and A.W.O. designed and performed experiments and contributed to the manuscript; T.B.S. performed experiments; C.L. scored tissue sections for GVHD histopathology and contributed to the manuscript; P.R. provided insight; G.D.G and J.L.M.F. conceived of and supervised all studies and contributed to the manuscript. E.G., A.W.O., G.D.G. and J.L.M.F. acknowledge stock ownership and consulting compensation from a corporation that has licensed certain commercial rights to Bz-423.

\section{References and Notes}

1. Jones RG, Thompson CB. Revving the engine: signal transduction fuels T cell activation. Immunity. 2007; 27:173-178. [PubMed: 17723208]

2. Vander Heiden MG, Cantley LC, Thompson CB. Understanding the Warburg effect: the metabolic requirements of cell proliferation. Science. 2009; 324:1029-1033. [PubMed: 19460998]

3. Voet, D.; Voet, JG. Biochemistry. 3. New York: J. Wiley \& Sons; 2004. p. xv-1591.

4. Cavazzana-Calvo M, Andre-Schmutz I, Dal Cortivo L, Neven B, Hacein-Bey-Abina S, Fischer A. Immune reconstitution after haematopoietic stem cell transplantation: obstacles and anticipated progress. Curr Opin Immunol. 2009; 21:544-548. [PubMed: 19766472]

5. Simsek T, Kocabas F, Zheng J, Deberardinis RJ, Mahmoud AI, Olson EN, Schneider JW, Zhang $\mathrm{CC}$, Sadek HA. The distinct metabolic profile of hematopoietic stem cells reflects their location in a hypoxic niche. Cell Stem Cell. 2010; 7:380-390. [PubMed: 20804973]

6. Cutler, C.; Antin, J. Manifestations and treatment of acute graft-versus-host disease. In: Appelbaum, FR.; Forman, S.; Negrin, RS.; Blume, K., editors. Thomas' Hematopoietic Cell Transplantation. 4. Vol. chap 86. Wiley-Blackwell; New Jersey: 2008.

7. Ferrara JL, Levine JE, Reddy P, Holler E. Graft-versus-host disease. Lancet. 2009; 373:1550-1561. [PubMed: 19282026]

8. Kuhnke A, Burmester GR, Krauss S, Buttgereit F. Bioenergetics of immune cells to assess rheumatic disease activity and efficacy of glucocorticoid treatment. Ann Rheum Dis. 2003; 62:133139. [PubMed: 12525382]

9. Wahl DR, Petersen B, Warner R, Richardson BC, Glick GD, Opipari AW. Characterization of the metabolic phenotype of chronically activated lymphocytes. Lupus. 2010 in press. 10.1177/0961203310373109

10. Blatt NB, Bednarski JJ, Warner RE, Leonetti F, Johnson KM, Boitano A, Yung R, Richardson BC, Johnson KJ, Ellman JA, Opipari AW Jr, Glick GD. Benzodiazepine-induced superoxide signals B cell apoptosis: mechanistic insight and potential therapeutic utility. J Clin Invest. 2002; 110:11231132. [PubMed: 12393848]

11. Sundberg TB, Swenson L, Wahl DR, Opipari AW Jr, Glick GD. Apoptotic signaling activated by modulation of the F0F1-ATPase: implications for selective killing of autoimmune lymphocytes. J Pharmacol Exp Ther. 2009; 331:437-444. [PubMed: 19706792]

12. Harris SI, Balaban RS, Mandel LJ. Oxygen consumption and cellular ion transport: evidence for adenosine triphosphate to $\mathrm{O} 2$ ratio near 6 in intact cell. Science. 1980; 208:1148-1150. [PubMed: 6246581]

13. Sariban-Sohraby S, Magrath IT, Balaban RS. Comparison of energy metabolism in human normal and neoplastic (Burkitt's lymphoma) lymphoid cells. Cancer Res. 1983; 43:4662-4664. [PubMed: 6883323]

14. Schieke SM, Phillips D, McCoy JP Jr, Aponte AM, Shen RF, Balaban RS, Finkel T. The mammalian target of rapamycin (mTOR) pathway regulates mitochondrial oxygen consumption and oxidative capacity. J Biol Chem. 2006; 281:27643-27652. [PubMed: 16847060] 
15. Maciver NJ, Jacobs SR, Wieman HL, Wofford JA, Coloff JL, Rathmell JC. Glucose metabolism in lymphocytes is a regulated process with significant effects on immune cell function and survival. $\mathrm{J}$ Leukoc Biol. 2008; 84:949-957. [PubMed: 18577716]

16. Zhao Y, Wieman HL, Jacobs SR, Rathmell JC. Mechanisms and methods in glucose metabolism and cell death. Methods Enzymol. 2008; 442:439-457. [PubMed: 18662583]

17. Puliaev R, Nguyen P, Finkelman FD, Via CS. Differential requirement for IFN-gamma in CTL maturation in acute murine graft-versus-host disease. J Immunol. 2004; 173:910-919. [PubMed: 15240678]

18. Brand KA, Hermfisse U. Aerobic glycolysis by proliferating cells: a protective strategy against reactive oxygen species. FASEB J. 1997; 11:388-395. [PubMed: 9141507]

19. Greiner EF, Guppy M, Brand K. Glucose is essential for proliferation and the glycolytic enzyme induction that provokes a transition to glycolytic energy production. J Biol Chem. 1994; 269:31484-31490. [PubMed: 7989314]

20. Brand MD. The efficiency and plasticity of mitochondrial energy transduction. Biochem Soc Trans. 2005; 33:897-904. [PubMed: 16246006]

21. Hill BG, Dranka BP, Zou L, Chatham JC, Darley-Usmar VM. Importance of the bioenergetic reserve capacity in response to cardiomyocyte stress induced by 4-hydroxynonenal. Biochem J. 2009; 424:99-107. [PubMed: 19740075]

22. Nicholls DG, Ward MW. Mitochondrial membrane potential and neuronal glutamate excitotoxicity: mortality and millivolts. Trends Neurosci. 2000; 23:166-174. [PubMed: 10717676]

23. Korshunov SS, Skulachev VP, Starkov AA. High protonic potential actuates a mechanism of production of reactive oxygen species in mitochondria. FEBS Lett. 1997; 416:15-18. [PubMed: 9369223]

24. Benov L, Sztejnberg L, Fridovich I. Critical evaluation of the use of hydroethidine as a measure of superoxide anion radical. Free Radic Biol Med. 1998; 25:826-831. [PubMed: 9823548]

25. Johnson KM, Chen X, Boitano A, Swenson L, Opipari AW Jr, Glick GD. Identification and validation of the mitochondrial F1F0-ATPase as the molecular target of the immunomodulatory benzodiazepine Bz-423. Chem Biol. 2005; 12:485-496. [PubMed: 15850986]

26. Shlomchik WD, Couzens MS, Tang CB, McNiff J, Robert ME, Liu J, Shlomchik MJ, Emerson SG. Prevention of graft versus host disease by inactivation of host antigen-presenting cells. Science. 1999; 285:412-415. [PubMed: 10411505]

27. Beilhack A, Schulz S, Baker J, Beilhack GF, Wieland CB, Herman EI, Baker EM, Cao YA, Contag $\mathrm{CH}$, Negrin RS. In vivo analyses of early events in acute graft-versus-host disease reveal sequential infiltration of T-cell subsets. Blood. 2005; 106:1113-1122. [PubMed: 15855275]

28. Bednarski JJ, Warner RE, Rao T, Leonetti F, Yung R, Richardson BC, Johnson KJ, Ellman JA, Opipari AW Jr, Glick GD. Attenuation of autoimmune disease in Fas-deficient mice by treatment with a cytotoxic benzodiazepine. Arthritis Rheum. 2003; 48:757-766. [PubMed: 12632430]

29. Caserta TM, Smith AN, Gultice AD, Reedy MA, Brown TL. Q-VD-OPh, a broad spectrum caspase inhibitor with potent antiapoptotic properties. Apoptosis. 2003; 8:345-352. [PubMed: 12815277]

30. Fox CJ, Hammerman PS, Thompson CB. Fuel feeds function: energy metabolism and the T-cell response. Nat Rev Immunol. 2005; 5:844-852. [PubMed: 16239903]

31. Vaughn AE, Deshmukh M. Glucose metabolism inhibits apoptosis in neurons and cancer cells by redox inactivation of cytochrome c. Nat Cell Biol. 2008; 10:1477-1483. [PubMed: 19029908]

32. Curis E, Nicolis I, Moinard C, Osowska S, Zerrouk N, Benazeth S, Cynober L. Almost all about citrulline in mammals. Amino Acids. 2005; 29:177-205. [PubMed: 16082501]

33. Nagy G, Koncz A, Fernandez D, Perl A. Nitric oxide, mitochondrial hyperpolarization, and T cell activation. Free Radic Biol Med. 2007; 42:1625-1631. [PubMed: 17462531]

34. Paczesny S, Choi SW, Ferrara JL. Acute graft-versus-host disease: new treatment strategies. Curr Opin Hematol. 2009; 16:427-436. [PubMed: 19812490]

35. Blatt NB, Boitano AE, Lyssiotis CA, Opipari AW Jr, Glick GD. Bz-423 superoxide signals apoptosis via selective activation of JNK, Bak and Bax. Free Radic Biol Med. 2008; 45:12321242. [PubMed: 18718527] 
36. Dai J, Weinberg RS, Waxman S, Jing Y. Malignant cells can be sensitized to undergo growth inhibition and apoptosis by arsenic trioxide through modulation of the glutathione redox system. Blood. 1999; 93:268-277. [PubMed: 9864170]

37. Morales MC, Perez-Yarza G, Nieto-Rementeria N, Boyano MD, Jangi M, Atencia R, Asumendi A. Intracellular glutathione levels determine cell sensitivity to apoptosis induced by the antineoplasic agent N-(4-hydroxyphenyl) retinamide. Anticancer Res. 2005; 25:1945-1951. [PubMed: 16158929]

38. Ramanathan B, Jan KY, Chen CH, Hour TC, Yu HJ, Pu YS. Resistance to paclitaxel is proportional to cellular total antioxidant capacity. Cancer Res. 2005; 65:8455-8460. [PubMed: 16166325]

39. Ashwell JD, Lu FW, Vacchio MS. Glucocorticoids in T cell development and function*. Annu Rev Immunol. 2000; 18:309-345. [PubMed: 10837061]

40. Jenkins MK, Schwartz RH, Pardoll DM. Effects of cyclosporine A on T cell development and clonal deletion. Science. 1988; 241:1655-1658. [PubMed: 3262237]

41. Araki K, Turner AP, Shaffer VO, Gangappa S, Keller SA, Bachmann MF, Larsen CP, Ahmed R. mTOR regulates memory CD8 T-cell differentiation. Nature. 2009; 460:108-112. [PubMed: 19543266]

42. Pearce EL, Walsh MC, Cejas PJ, Harms GM, Shen H, Wang LS, Jones RG, Choi Y. Enhancing CD8 T-cell memory by modulating fatty acid metabolism. Nature. 2009; 460:103-107. [PubMed: 19494812]

43. Hill GR, Crawford JM, Cooke KR, Brinson YS, Pan L, Ferrara JL. Total body irradiation and acute graft-versus-host disease: the role of gastrointestinal damage and inflammatory cytokines. Blood. 1997; 90:3204-3213. [PubMed: 9376604]

44. Gatza E, Rogers CE, Clouthier SG, Lowler KP, Tawara I, Liu C, Reddy P, Ferrara JL. Extracorporeal photopheresis reverses experimental graft-versus-host disease through regulatory $\mathrm{T}$ cells. Blood. 2008; 112:1515-1521. [PubMed: 18411417] 

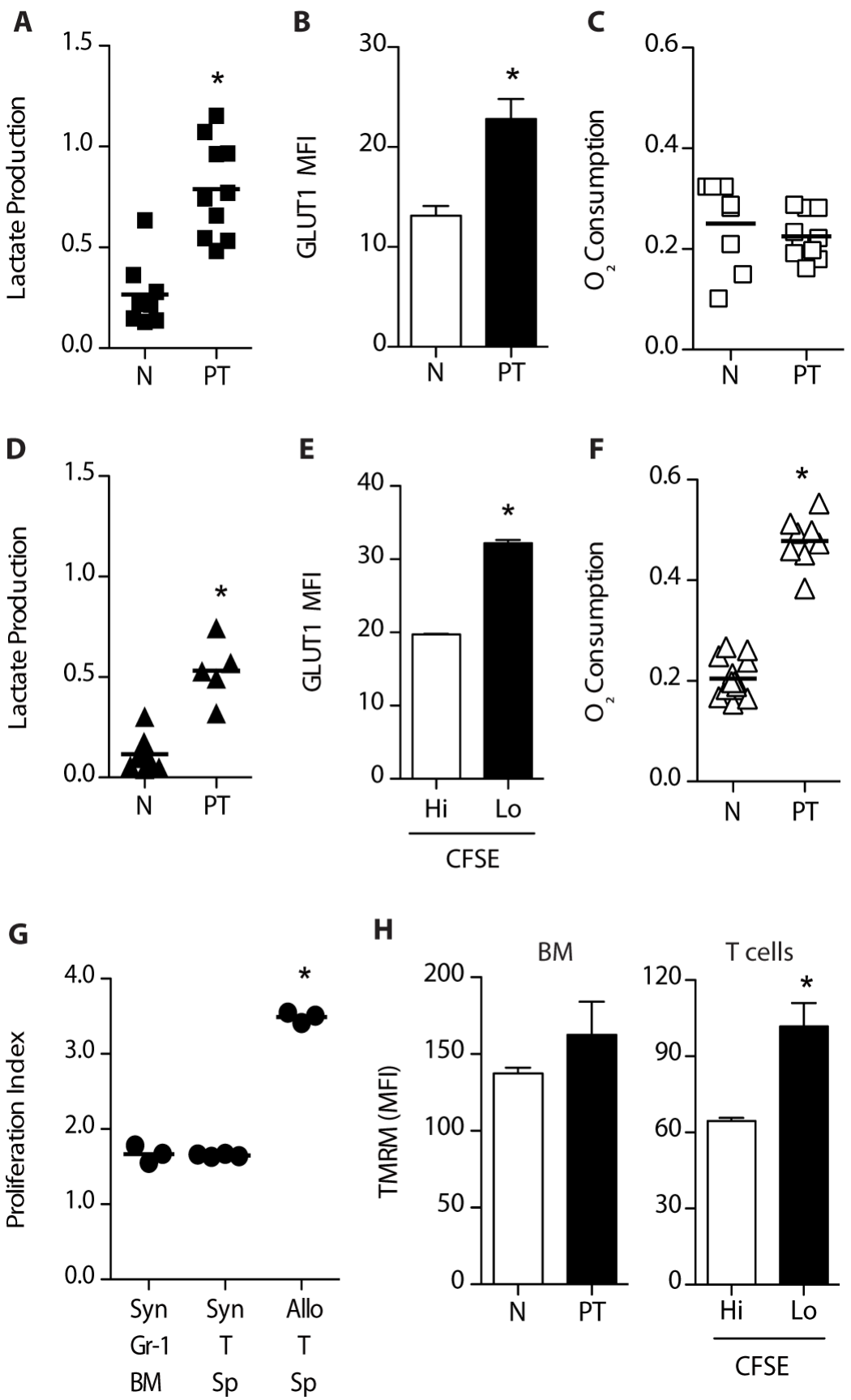

Figure 1.

Bioenergetics of T cells mediating GVHD. (A-C) Lethally irradiated B6-Ly5.2 mice received $\mathrm{BM}\left(5.0 \times 10^{6}\right.$ cells $)$ from B6 donors. Analysis was performed on BM cells taken from mice 8-9 days post transplant (PT) and from naïve B6 mice $(\mathrm{N})$. (A) Lactate production (nMoles lactate/(minute $\times 10^{6}$ cells)), (B) GLUT1 expression and (C) oxygen consumption (nMoles $\mathrm{O}_{2} /\left(\right.$ minute $\times 10^{6}$ cells)) by BM cells ( $\mathrm{n}=10 /$ group). (D) B6-Thy1.1 splenocytes $\left(50 \times 10^{6}\right)$ were injected into allogeneic $\mathrm{B} 6 \mathrm{D} 2 \mathrm{~F} 1$ recipients. Lactate production was measured in alloreactive donor T cells 7 days later $(\mathrm{PT})$ and in naïve B6 mice $(\mathrm{N})$. (E) CFSE-labeled B6-Ly5.2 splenocytes $\left(50 \times 10^{6}\right)$ were injected into allogeneic B6D2F1 recipients. GLUT1 expression was determined by flow cytometry in undivided (CFSE ${ }^{\text {hi }}$ ) or 
divided (CFSE ${ }^{l o}$ ) donor T cells ( $\mathrm{n}=3$ /group). (F) Mice were transplanted as in (D) and oxygen consumption by alloreactive donor $T$ cells was measured 7 days later. $(\mathrm{G})$ Proliferation indices. CFSE-labeled B6 or B6-Ly5.2 donor cells were transplanted into B6Ly5.2 (Syn) or B6D2F1 (Allo) recipients. Donor cells were identified based on CD45.1 or CD45.2 expression on day 3 post transplant in the $\mathrm{BM}$ or spleen (Sp) and gated as $\mathrm{Gr}-1^{+}$ $(\mathrm{Gr}-1)$ or $\mathrm{CD} 4 / 8^{+}(\mathrm{T})$. (H) Animals were transplanted as in (A) and (E). TMRM staining 7-8 days after transplant (BM, $n=8 /$ group) or on day 4 after transplant ( $T$ cells, $n=6 /$ group). Bar plots represent mean \pm SEM, ${ }^{*} \mathrm{p}<0.005$. 
A

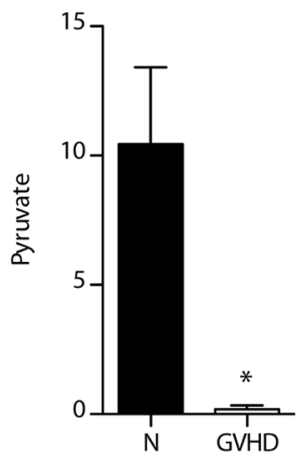

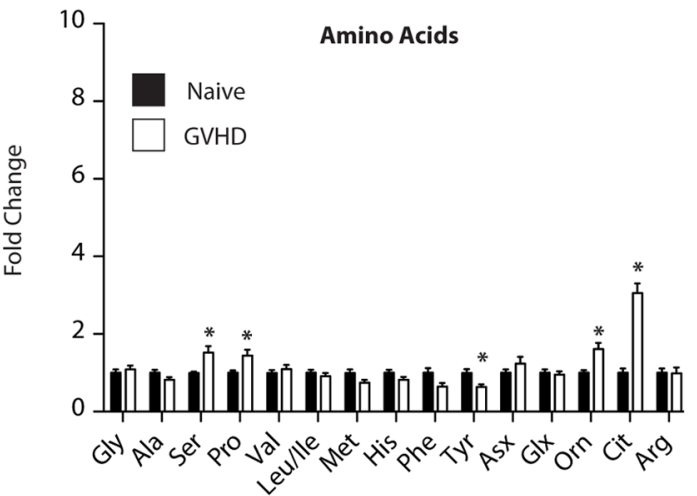

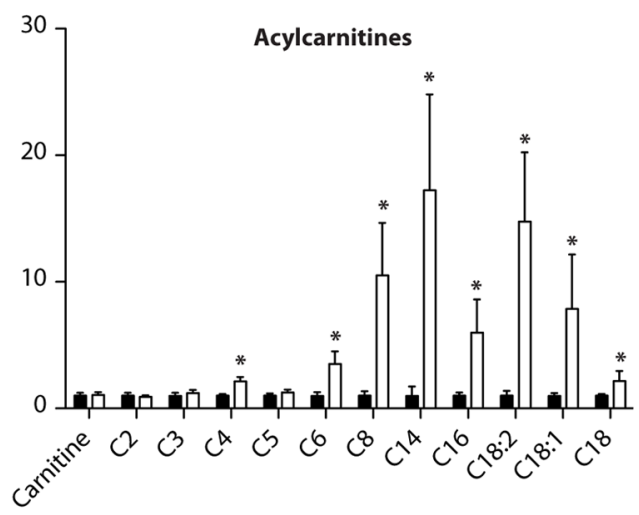

Figure 2.

T cells mediating GVHD have decreased intracellular pyruvate and increased intracellular acylcarnitines. B6-Thy1.1 splenocytes $\left(50 \times 10^{6}\right)$ were injected into allogeneic B6D2F1 recipients as in Figure 1D. Donor Thy $1.1^{+} \mathrm{T}$ cells were isolated from spleens of recipient B6D2F1 (GVHD) mice 7 days later and compared to Thy $1.1^{+} \mathrm{T}$ cells isolated from naive B6-Thy1.1 mice. (A) Intracellular pyruvate levels (pMoles pyruvate/ $\mu$ g protein, $n=5 / g r o u p$ ). Bars represent mean \pm SEM; white vs black bar, * ${ }^{2}<0.006$. (B-C) T cell pellets were sonicated in water and snap frozen in liquid nitrogen prior to metabolomic analysis. Each pellet contained T cells from 4-6 transplanted mice with GVHD or 2 naïve donor mice. Bar plots represent means \pm SEM ( $n=8-12$ naïve donors or $n=16-24$ mice with GVHD). Amino acid (B) and acylcarnitine (C) concentrations in GVHD cells are expressed as the folddifference from naïve donor cells, determined on the basis of analyte concentrations and normalized to the protein content of each sample; white vs black bars, ${ }^{*} \mathrm{p}<0.05$. Acylcarnitine species abbreviated as follows: C2:0, Acetylcarnitine; C3:0, Propionylcarnitine; C4:0, Butyrylcarnitine; C5:0, Valerylcarnitine; C6:0, Hexanoylcarnitine; C8:0, Octanoylcarnitine; C14:0, Myristoylcarnitine; C16:0, Palmitoylcarnitine; C18:2, Linoleoylcarnitine; C18:1, Oleoylcarnitine; C18:0, Stearoylcarnitine. 
A

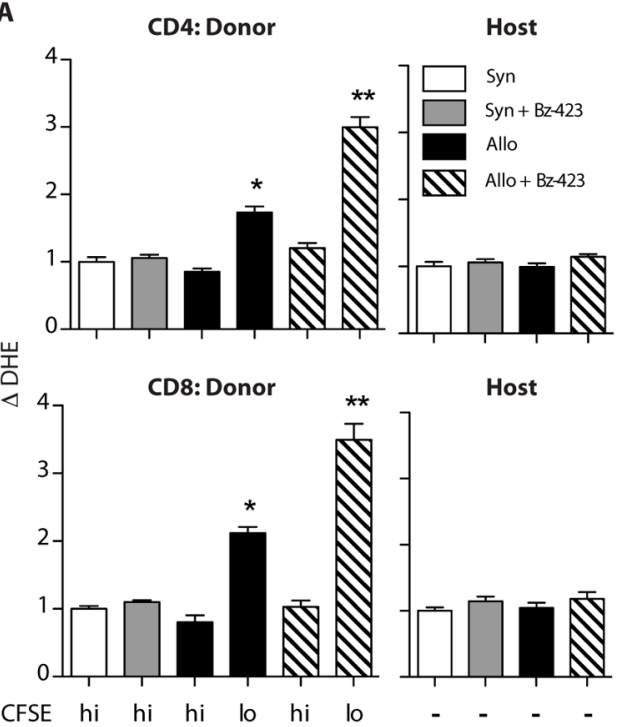

B

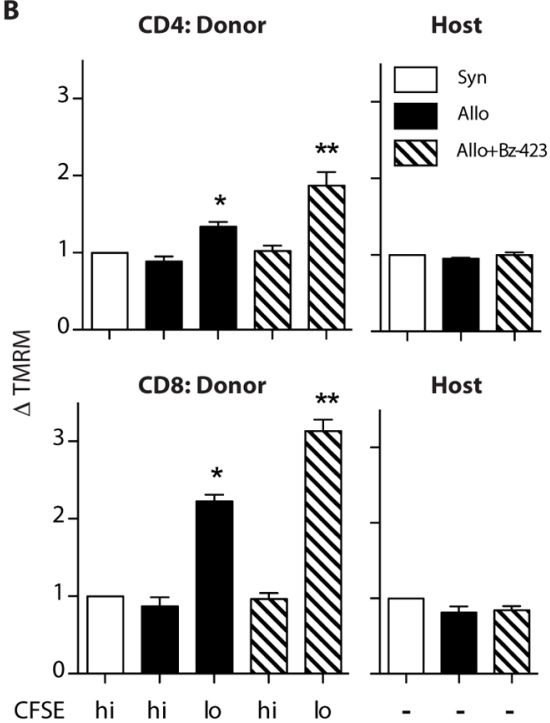

$\mathbf{E}$

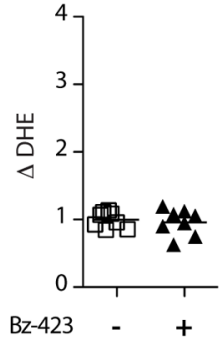

$\mathbf{F}$

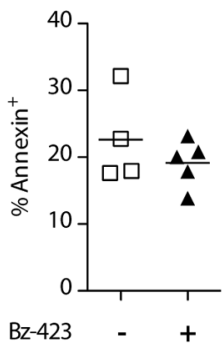

Figure 3.

Bz-423 increases superoxide, $\Delta \Psi_{\mathrm{m}}$ and apoptosis in dividing donor $\mathrm{T}$ cells but not $\mathrm{BM}$ cells. (A-B) Syngeneic (B6) and allogeneic (B6D2F1) recipient mice were infused with $50 \times 10^{6}$ CFSE-labeled splenocytes from B6-Ly5.2 donor mice on day 0 followed by a single injection of Bz-423 or vehicle on day 4. (A) DHE and (B) TMRM staining of gated donor $\left(\mathrm{CD} 45.1^{+} \mathrm{CFSE}^{\mathrm{lo} / \mathrm{hi}} \mathrm{CD}^{+}\right.$or $\left.\mathrm{CD}^{+}\right)$or host $\left(\mathrm{CD} 45.2^{+} \mathrm{CD}^{+}\right.$or $\left.\mathrm{CD} 8^{+}\right)$populations plotted as fold-increase in staining above donor and host cell staining in syngeneic controls $(n=6-8 /$ group); *p $₫ 0.001$, **p $₫ 0.02$. (C) Annexin-V staining of donor $\mathrm{CD}^{+}$and $\mathrm{CD} 8^{+}$cells from allogeneic recipient mice 7 days after donor cell infusion and 6 hours after a single Bz-423 or control injection ( $n=12 /$ group); *p $₫$ 0.001. (D-F) Lethally irradiated B6-Ly5.2 mice were transplanted with syngeneic B6 BM $\left(5.0 \times 10^{6}\right)$ and given a single injection of vehicle or Bz-423 8 days after transplant. (D) DHE and (E) TMRM staining were analyzed 2 hours following injections. Data were plotted as fold-increase in staining above vehicle-treated controls. (F) Annexin-V staining was measured 6 hours after injection of vehicle or Bz-423. Data in all bar plots are means \pm SEM pooled from 2-4 independent experiments. 
A

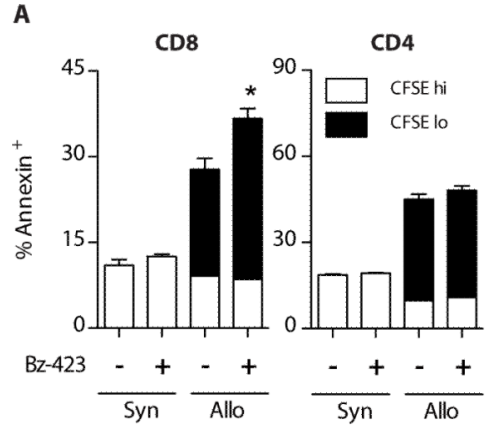

B

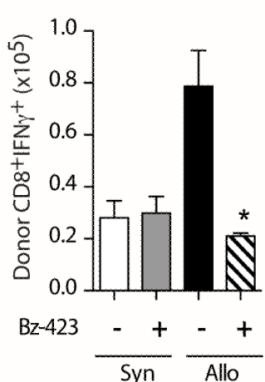

c

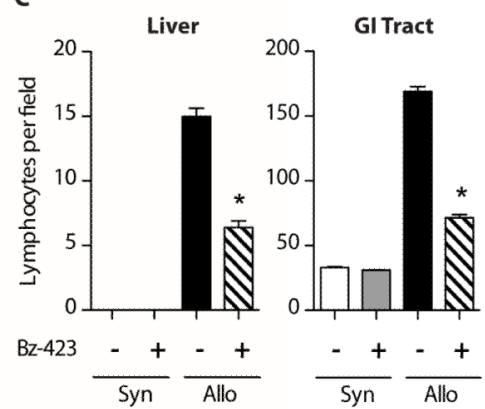

D
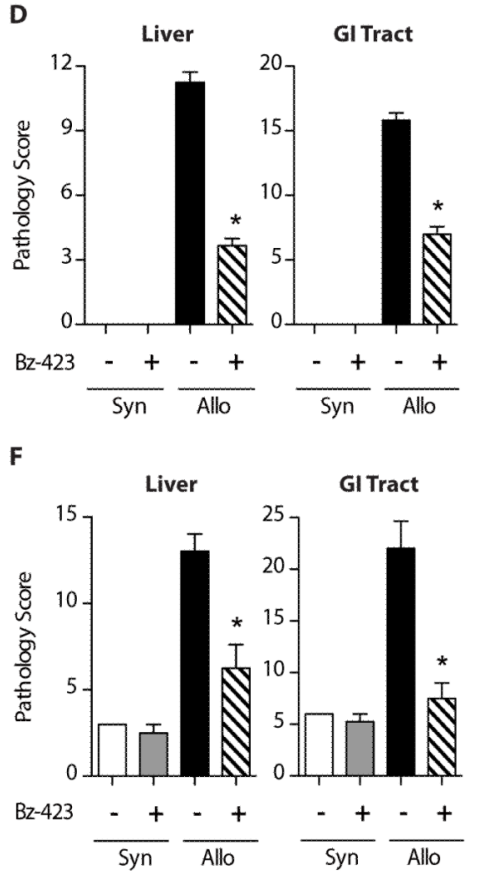

E
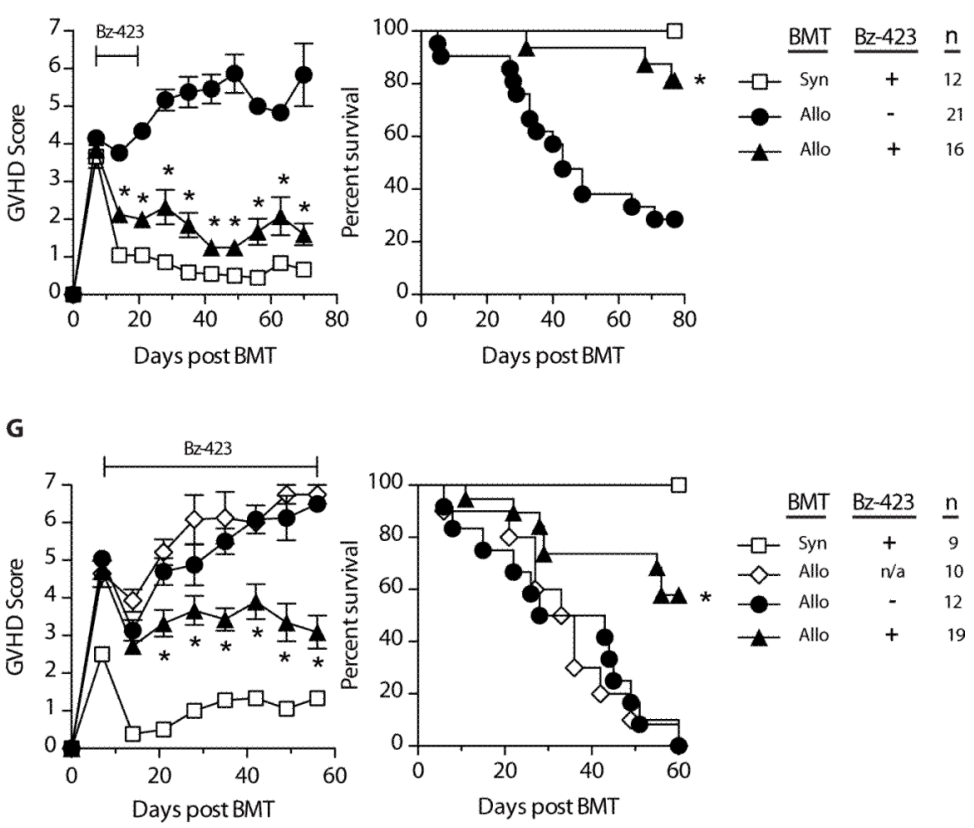

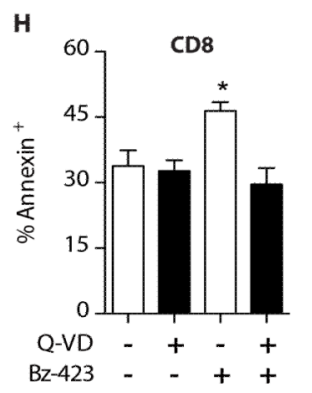

I
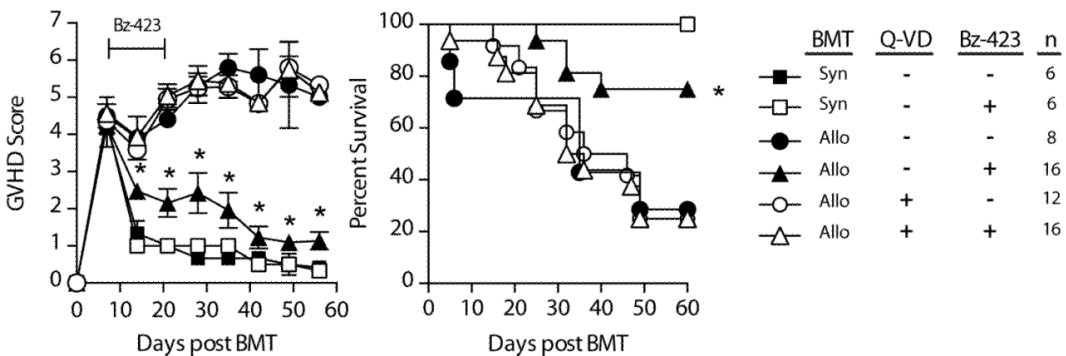

Figure 4.

Bz-423 induces apoptosis in donor T cells and reduces GVHD after BMT. (A) B6-Ly5.2 mice received CFSE-labeled CD90 ${ }^{+} \mathrm{T}$ cells from B6 (syngeneic) or $\mathrm{C} 3 \mathrm{H} . \mathrm{SW}$ (allogeneic) donors on day 0 and one $\mathrm{Bz}-423$ injection 4 days later. Annexin- $\mathrm{V}$ staining of donor $\mathrm{CFSE}^{\text {hi/lo }} \mathrm{CD}^{+}$and $\mathrm{CD}^{+}$cells 6 hours after Bz-423 injection (n=6/group); $\mathrm{CFSE}^{\text {lo }} \mathrm{CD}^{+}$ $\mathrm{Bz}-423$ vs vehicle, ${ }^{*} \mathrm{p}=0.04$. (B-E) B6-Ly5.2 mice received BM and CD90 ${ }^{+} \mathrm{T}$ cells from $\mathrm{B} 6$ or C3H.SW donors on day 0 and 6 doses of Bz-423 beginning on day 7. (B) Donor $\mathrm{CD} 8^{+} \mathrm{IFN}-\gamma^{+}(\mathrm{n}=6 /$ group$)$ in the spleen on day 21 ; hatched vs black bar, ${ }^{*} \mathrm{p}=0.002$. (C) Lymphocyte infiltration in the liver and GI tract 42 days after BMT ( $n=3-5 /$ group, 10 high power fields counted/animal/site); hatched vs black bars, $* \mathrm{p}<0.03$. (D) Histopathology 
scores 42 days after BMT (n=6/group); hatched vs black bars, * $\mathrm{p}<0.03$. (E) GVHD scores and survival; triangles vs circles, ${ }^{*} \mathrm{p}<0.005$. (F-G) B6 mice received BM and CD $90^{+} \mathrm{T}$ cells from syngeneic B6-Ly5.2 or allogeneic Balb/c donors on day 0 and were injected with Bz-423 or vehicle $3 \mathrm{x} /$ week for 7 weeks beginning on day 7. (F) Histopathology scores 56 days after BMT (n=6-8/group); hatched vs black bars, *p<0.03. (G) GVHD scores and survival; triangles vs circles, ${ }^{*} \mathrm{p}<0.02 ; \mathrm{n} / \mathrm{a}$, received neither vehicle nor Bz-423. $(\mathrm{H})$ Mice were transplanted as in (B-E) and injected with Q-VD- OPH or DMSO control on days 5-7. $\mathrm{Bz}-423$ or vehicle was injected 1 hour following the last Q-VD-OPH injection. Annexin-V staining of allogeneic donor $\mathrm{CFSE}^{\mathrm{lo}} \mathrm{CD} 8^{+}$cells was determined 6 hours later (n=6/group); * vs all other groups, $\mathrm{p} \unlhd$ (0.03. (I) Mice transplanted and injected with Bz-423 as in (B-E) were also injected with Q-VD-OPH or DMSO control on days 5-19. GVHD scores and survival; black triangles vs open triangles or black circles, *p $₫$ ().01. All data are means \pm SEM pooled from at least 2 independent experiments. 